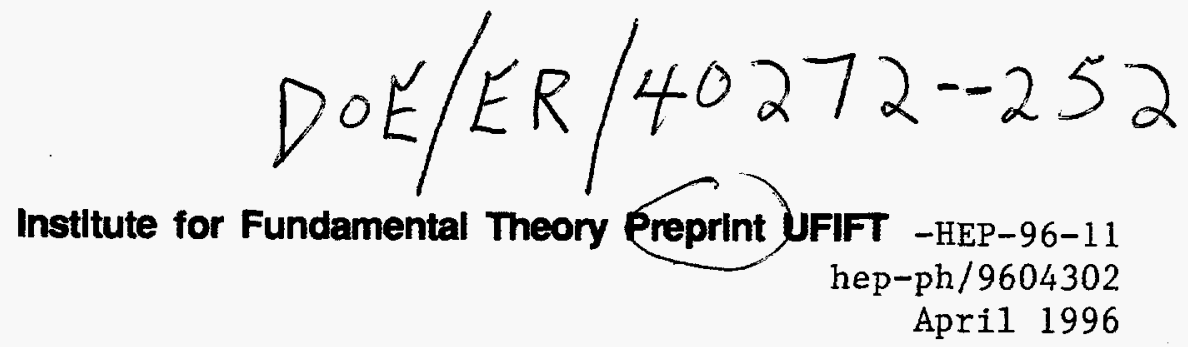

\title{
Leptophobic $Z^{\prime}$
}

\section{From Superstring Derived NGEEIVED}

\author{
Alon E. Faraggi ${ }^{1 *}$ and Manuel Masip MA 171996 \\ 1 Institute For Fundamental Theory, \\ University of Florida, Gainesville, FL 32611 USA \\ 2 Departamento de Física Teórica y del Cosmos, \\ Universidad de Granada, 18071 Granada, Spain
}

e STid

\begin{abstract}
It was recently suggested that the reported anomalies in $R_{b}$ and $R_{c}$ can be interpreted as the effect of a heavy vector boson that couples to quarks and is universally decoupled from leptons. We examine how an extra gauge boson with this property can arise from superstring derived models. In a specific three generation model we show that the $U(1)_{B-L}$ symmetry combines with the horizontal flavor symmetries to form a universal leptophobic $U(1)$ symmetry. In our model there is an enhancement of the color gauge group from twisted sectors. The enhancement occurs after the breaking of the unifying gauge symmetry by "Wilson lines". The leptophobic $U(1)$ symmetry then becomes a generator of the color $S U(4)$ gauge group. We examine how similar symmetries may appear in other string models without the enhancement. We propose that if the current LEP anomalies persist it may be evidence for a certain class of un-unified superstring models.
\end{abstract}

*E-mail address: faraggi@phys.ufl.edu

${ }^{\dagger} \mathrm{E}$-mail address: masip@ugr.es 



\section{DISCLAIMER}

Portions of this document may be illegible in electronic image products. Images are produced from the best available original document. 

Over the past few years LEP, SLC and the Tevatron experiments provided impressive confirmation of the Standard Model of particle physics and its gauge symmetry structure. Recently, however, there has been accumulating evidence at LEP that indicates deviation from the Standard Model predictions in the hadronic partial width at the $Z$-boson peak [1], which is commonly referred to as the $R_{b}-R_{c}$ crisis.

It was recently suggested by several groups [2] that the discrepancy between the predicted and measured values for the decay of the $Z$-boson to $b$ and $c$-quarks could be explained by an additional heavy gauge boson that couples to quarks but is universally decoupled from leptons. If this interpretation of the data is correct it will have profound implication on attempts to understand the origin of the gauge and matter structure of the Standard Model. It might for example invalidate the traditional approaches to embed the Standard Model in a simple Grand Unified Group as those in their nature unify the interactions between quarks and leptons.

In superstring models one also traditionally starts with an underlying unifying gauge group which is then broken to the Standard Model by using string and field theoretic symmetry breaking mechanisms. However, as the rank of the gauge group in string models is larger than those which are used in Grand Unified Theories, one can contemplate the possibility that a particular combination of the additional $U(1)$ generators in the four dimensional Cartan subalgebra will combine to form a leptophobic $U(1)$. Moreover, in a generic level one string model, massless states that produce the Standard Model representations, in general, must be charged with respect to additional $U(1)$ or discrete symmetries. As the assignment of charges to the quarks and lepton depend on the specific compactification, it is difficult to envision how a generic compactification will produce the universal charge assignment which is needed. Furthermore, the charges of the quarks and leptons under the additional $U(1)$ symmetries of the four dimensional gauge group depend on specific patterns of symmetry breaking in the string models. Therefore, if such a leptophobic $U(1)$ symmetry is produced it might be a peculiar accident of a particular string model or perhaps of a class of string models.

In this paper, we examine how a leptophobic $U(1)$ can arise from superstring 
derived models. The leptophobia of the $U(1)$ symmetry is obtained by combining the $U(1)_{B-L}$ generator with a combination of the flavor $U(1)$ symmetries. We present an explicit superstring derived model which gives rise to a universal leptophobic $U(1)$ symmetry. The leptophobic $U(1)$ symmetry is obtained in a class of superstring derived standard-like models [3] due to a combination of the $U(1)_{B-L}$ symmetry, which is embedded in $S O(10)$, plus a combination of additional $U(1)$ symmetries. These additional $U(1)$ symmetries compensate the lepton number in $U(1)_{B-L}$ and the resulting $U(1)$ therefore becomes a gauged baryon number. In the specific model that we study in some detail the gauge symmetry is enhanced due to gauge bosons from twisted sectors. The color $S U(3)$ gauge group is enhanced to $S U(4)$, and $U(1)_{B}$ is the $U(1)$ in the decomposition $S U(4)_{C} \rightarrow S U(3)_{C} \times U(1)_{B}$. Due to a symmetry between the three chiral generation, we argue that leptophobic $U(1)$ symmetries may in fact be common in this class of superstring compactification, without further enhancement of the gauge group.

The superstring models that we discuss are constructed in the free fermionic formulation [4]. In this formulation a model is constructed by choosing a consistent set of boundary condition basis vectors. The basis vectors, $b_{k}$, span a finite additive group $\Xi=\sum_{k} n_{k} b_{k}$ where $n_{k}=0, \cdots, N_{z_{k}}-1$. The physical massless states in the Hilbert space of a given sector $\alpha \in \Xi$, are obtained by acting on the vacuum with bosonic and fermionic operators and by applying the generalized GSO projections. The $U(1)$ charges, $Q(f)$, with respect to the unbroken Cartan generators of the four dimensional gauge group, which are in one to one correspondence with the $U(1)$ currents $f^{*} f$ for each complex fermion $\mathrm{f}$, are given by:

$$
Q(f)=\frac{1}{2} \alpha(f)+F(f),
$$

where $\alpha(f)$ is the boundary condition of the world-sheet fermion $f$ in the sector $\alpha$, and $F_{\alpha}(f)$ is a fermion number operator counting each mode of $f$ once (and if $f$ is complex, $f^{*}$ minus once). For periodic fermions, $\alpha(f)=1$, the vacuum is a spinor in order to represent the Clifford algebra of the corresponding zero modes. For each periodic complex fermion $f$ there are two degenerate vacua $|+\rangle,|-\rangle$, annihilated by the zero modes $f_{0}$ and $f_{0}{ }^{*}$ and with fermion numbers $F(f)=0,-1$, respectively. 
The realistic models in the free fermionic formulation are generated by a basis of boundary condition vectors for all world-sheet fermions $[5,3,6,7,8,9,10]$. The basis is constructed in two stages. The first stage consist of the NAHE set $[5,3]$, which is a set of five boundary condition basis vectors, $\left\{1, S, b_{1}, b_{2}, b_{3}\right\}$. The gauge group after the NAHE set is $S O(10) \times S O(6)^{3} \times E_{8}$ with $N=1$ space-time supersymmetry. The vector $S$ is the supersymmetry generator and the superpartners of the states from a given sector $\alpha$ are obtained from the sector $S+\alpha$. The space-time vector bosons that generate the gauge group arise from the Neveu-Schwarz sector and from the sector $1+b_{1}+b_{2}+b_{3}$. The Neveu-Schwarz sector produces the generators of $S O(10) \times S O(6)^{3} \times S O(16)$. The sector $1+b_{1}+b_{2}+b_{3}$ produces the spinorial 128 of $S O(16)$ and completes the hidden gauge group to $E_{8}$. The vectors $b_{1}, b_{2}$ and $b_{3}$ produce 48 spinorial 16 of $S O(10)$, sixteen from each sector $b_{1}, b_{2}$ and $b_{3}$. The vacuum of these sectors contains eight periodic fermions. Five of those periodic fermions produce the charges under the $S O(10)$ group, while the remaining three periodic fermions generate charges with respect to the flavor symmetries. Each of the sectors $b_{1}, b_{2}$ and $b_{3}$ is charged with respect to a different set of flavor quantum numbers, $S O(6)_{1,2,3}$.

The NAHE set divides the 44 right-moving and 20 left-moving real internal fermions in the following way: $\bar{\psi}^{1, \cdots, 5}$ are complex and produce the observable $S O(10)$ symmetry; $\bar{\phi}^{1, \cdots, 8}$ are complex and produce the hidden $E_{8}$ gauge group; $\left\{\bar{\eta}^{1}, \bar{y}^{3, \cdots, 6}\right\}$, $\left\{\bar{\eta}^{2}, \bar{y}^{1,2}, \bar{\omega}^{5,6}\right\},\left\{\bar{\eta}^{3}, \bar{\omega}^{1, \cdots, 4}\right\}$ give rise to the three horizontal $S O(6)$ symmetries. The left-moving $\{y, \omega\}$ states are divided into, $\left\{y^{3, \cdots, 6}\right\},\left\{y^{1,2}, \omega^{5,6}\right\},\left\{\omega^{1, \cdots, 4}\right\}$. The leftmoving $\chi^{12}, \chi^{34}, \chi^{56}$ states carry the supersymmetry charges. Each sector $b_{1}, b_{2}$ and $b_{3}$ carries periodic boundary conditions under $\left(\psi^{\mu} \mid \bar{\psi}^{1, \cdots, 5}\right)$ and one of the three groups: $\left(\chi_{12},\left\{y^{3, \cdots, 6} \mid \bar{y}^{3, \cdots 6}\right\}, \bar{\eta}^{1}\right),\left(\chi_{34},\left\{y^{1,2}, \omega^{5,6} \mid \bar{y}^{1,2} \bar{\omega}^{5,6}\right\}, \bar{\eta}^{2}\right),\left(\chi_{56},\left\{\omega^{1, \cdots, 4} \mid \bar{\omega}^{1, \cdots 4}\right\}, \bar{\eta}^{3}\right)$.

The division of the internal fermions is a reflection of the underlying $Z_{2} \times Z_{2}$ orbifold compactification [11]. The Neveu-Schwarz sector corresponds to the untwisted sector, and the sectors $b_{1}, b_{2}$ and $b_{3}$ correspond to the three twisted sectors of the $Z_{2} \times Z_{2}$ orbifold models. At this level there is a discrete $S_{3}$ permutation symmetry between the three sectors $b_{1}, b_{2}$ and $b_{3}$. This permutation symmetry arises due to the 
symmetry of the NAHE set and may be essential for the universality of the leptophobic $U(1)$ symmetry. Because of the underlying $Z_{2} \times Z_{2}$ orbifold compactification, each of the chiral generations from the sectors $b_{1}, b_{2}$ and $b_{3}$ is charged with respect to a different set of flavor charges.

The second stage of the basis construction consist of adding three additional basis vectors to the NAHE set. The three additional basis vectors correspond to "Wilson lines" in the orbifold formulation. Three additional vectors are needed to. reduce the number of generations to three, one from each sector $b_{1}, b_{2}$ and $b_{3}$. One specific example is given in table 1 . The choice of boundary conditions to the set of real internal fermions $\{y, \omega \mid \bar{y}, \bar{\omega}\}^{1, \cdots, 6}$ determines the low energy properties, like the number of generations, Higgs doublet-triplet splitting and Yukawa couplings.

The final gauge group arises as follows. The NS sector produces the generators of $S U(3)_{C} \times S U(2)_{L} \times U(1)_{C} \times U(1)_{L} \times U(1)_{1,2,3} \times U(1)_{4,5,6} \times S U(3) \times S O(4) \times U(1)_{H} \times$ $U(1)_{7,8,9}$. The $S O(10)$ symmetry is broken to $S U(3)_{C} \times U(1)_{C} \times S U(2)_{L} \times U(1)_{L}{ }^{*}$, where

$$
\begin{aligned}
& U(1)_{C}=\operatorname{Tr} U(3)_{C} \Rightarrow Q_{C}=\sum_{i=1}^{3} Q\left(\bar{\psi}^{i}\right), \\
& U(1)_{L} \quad=\operatorname{Tr} U(2)_{L} \Rightarrow Q_{L}=\sum_{i=4}^{5} Q\left(\bar{\psi}^{i}\right) .
\end{aligned}
$$

The flavor $S O(6)^{3}$ symmetries are broken to $U(1)^{3+n}$ with $(n=0, \cdots, 6)$. The first three, denoted by $U(1)_{r_{j}}$, arise from the world-sheet currents $\bar{\eta}^{j} \bar{\eta}^{j^{*}}(j=1,2,3)$. These three $U(1)$ symmetries are present in all the three generation free fermionic models which use the NAHE set. Additional horizontal $U(1)$ symmetries, denoted by $U(1)_{r_{j}}(j=4,5, \ldots)$, arise by pairing two real fermions from the sets $\left\{\bar{y}^{3, \cdots, 6}\right\}$, $\left\{\bar{y}^{1,2}, \bar{\omega}^{5,6}\right\}$, and $\left\{\bar{\omega}^{1, \cdots, 4}\right\}$. The final observable gauge group depends on the number of such pairings. In the model of table 1 there are three such pairings, $\bar{y}^{3} \bar{y}^{6}, \bar{y}^{1} \bar{\omega}^{5}$ and $\bar{\omega}^{2} \bar{\omega}^{4}$, which generate three additional $U(1)$ symmetries, denoted by $U(1)_{r_{4,5,6}}$. It is important to note that the existence of these three additional $U(1)$ currents is correlated with a superstringy doublet-triplet splitting mechanism [12]. Due to these

$$
{ }^{*} U(1)_{C}=\frac{3}{2} U(1)_{B-L} \text { and } U(1)_{L}=2 U(1)_{T_{B_{R}}} .
$$


extra $U(1)$ symmetries the color triplets from the NS sector are projected out of the spectrum by the GSO projections while the electroweak doublets remain in the light spectrum. The remaining $U(1)$ generators are

$$
U(1)_{H}=\operatorname{Tr} U(3)_{H} \Rightarrow Q_{H}=\sum_{i=5}^{7} Q\left(\bar{\phi}^{i}\right)
$$

and $U(1)_{7,8,9}$, which arise from the world-sheet currents $\bar{\phi}^{1} \bar{\phi}^{1^{*}}, \bar{\phi}^{2} \bar{\phi}^{2^{*}}, \bar{\phi}^{8} \bar{\phi}^{8^{*}}$, respectively. The sector $1+b_{1}+b_{2}+b_{3}$ produces the representations $(3,2)_{-5} \oplus(\overline{3}, 2)_{5}$ and $2_{-3} \oplus 2_{3}$ of $S U(3) \times S U(2)_{r} \times U(1)_{h 5}$ and $S U(2)_{\ell} \times U(1)_{h 3}$ respectively, where $S U(2)_{r} \times S U(2)_{\ell}$ are the two $S U(2)$ 's in the isomorphism $S O(4) \sim S U(2)_{r} \times S U(2)_{\ell}$. Thus, the $E_{8}$ symmetry reduces to $S U(5) \times S U(3) \times U(1)^{2}$. The $U(1)^{\prime}$ 's in $S U(5)$ and $S U(3)$ are given by $U(1)_{h 5}=-3 U_{7}+3 U_{8}+U_{H}-3 U_{9}$ and $U(1)_{h 3}=U_{7}+U_{8}+U_{H}+U_{9}$ respectively. The remaining $U(1)$ symmetries in the hidden sector, $U(1)_{7^{\prime}}$ and $U(1)_{8^{\prime}}$, correspond to the world-sheet currents $\bar{\phi}^{1} \bar{\phi}^{1^{*}}-\bar{\phi}^{8} \bar{\phi}^{8^{*}}$ and $-2 \bar{\phi}^{j} \bar{\phi}^{j^{*}}+\bar{\phi}^{1} \bar{\phi}^{1^{*}}+4 \bar{\phi}^{2} \bar{\phi}^{2^{*}}+$ $\bar{\phi}^{8} \bar{\phi}^{8^{*}}$ respectively, where summation on $j=5, \cdots, 7$ is implied.

For some choices of the additional basis vectors that extend the NAHE set, there may exist a combination

$$
X=n_{\alpha} \alpha+n_{\beta} \beta+n_{\gamma} \gamma
$$

for which $X_{L} \cdot X_{L}=0$ and $X_{R} \cdot X_{R} \neq 0$. Such a combination may produce additional space-time vector bosons, depending on the choice of GSO phases. In the model of table 1 additional space-time vector bosons are obtained from the sector $1+\alpha+2 \gamma$ [10]. The model of table 1 differs from the model of Ref. [10] by a change of a GSO phase

$$
c\left(\begin{array}{l}
\gamma \\
1
\end{array}\right)=-1 \rightarrow c\left(\begin{array}{l}
\gamma \\
1
\end{array}\right)=+1
$$

In the model of table 1 , the sector $1+\alpha+2 \gamma$ produces six additional space-time vector bosons, which are triplets of $S U(3)_{C}$ and carry $U(1)$ charges. One combination of the $U(1)$ symmetries

$$
U(1)_{B}=\frac{1}{3} U_{C}-\left(U_{r_{4}}+U_{r_{5}}+U_{r_{6}}\right)-U_{7^{\prime}}
$$

is the $U(1)$ generator of the enhanced color $S U(4)$ symmetry. The six space-time vector bosons from the sector $1+\alpha+2 \gamma$ complete the adjoint representation of the 
gauge group. The remaining orthogonal $U(1)$ combinations are

$$
\begin{aligned}
U_{C^{\prime}} & =U_{C}+\frac{1}{2} U_{7^{\prime}}, \\
U_{4^{\prime}} & =U_{4}-U_{5}, \\
U_{5^{\prime}} & =U_{4}+U_{5}-2 U_{6}, \\
U_{7^{\prime \prime}} & =U_{C}+\frac{7}{3}\left(U_{4}+U_{5}+U_{6}\right)-3 U_{7^{\prime}} .
\end{aligned}
$$

The full massless spectrum now transforms under the final gauge group, $S U(4)_{C} \times$ $S U(2)_{L} \times U(1)_{C^{\prime}} \times U(1)_{L} \times U(1)_{1,2,3} \times U(1)_{4^{\prime}} \times U(1)_{5^{\prime}} \times U(1)_{7^{\prime \prime}} \times U(1)_{8}$. The weak hypercharge is given by $U(1)_{Y}=1 / 3 U(1)_{C^{\prime}}+1 / 2 U(1)_{L}$. The Neveu-Schwarz sector gives, in addition to the graviton, dilaton, antisymmetric sector and spin 1 gauge bosons, three pairs of electroweak doublets, three pairs of $S O(10)$ singlets with $U(1)_{1,2,3}$ charges and three singlets of the entire four dimensional gauge group. The sector $S+b_{1}+b_{2}+\alpha+\beta$ produces two pairs of electroweak doublets and four pairs of $S O(10)$ singlets with $U(1)_{1,2,3}$ charges. The quantum numbers of the massless states from these two sectors are the same as those that are given in Ref. [10].

The states from the sectors $b_{j} \oplus 1+\alpha+2 \gamma(j=1,2,3)$ produce the three light generations. The states from these sectors and their decomposition under the entire gauge group are shown in table 2. The leptons are singlets of the color $S U(4)$ gauge group and the $U(1)_{B}$ symmetry, Eq. 6 becomes the gauged leptophobic $U(1)$ symmetry. The remaining massless states and their quantum numbers are given in table 2.

We observe that the leptophobia of the $U(1)_{B}$ symmetry is obtained from a combination of $U(1)_{B-L}$ plus the three flavor symmetries $U(1)_{r_{4,5,6}}$. The $Q_{C}$ charges of the leptons from each of the sectors $b_{1,2,3}$ are canceled by their charges under the flavor symmetries $U(1)_{r, 5,6}$. Miraculously, the charges of the leptons under the flavor $U(1)$ symmetries are such that the cancelation occurs for all the leptons, in all the sectors. Thus, the leptophobic $U(1)$ symmetry is generation blind.

The massless spectrum of the string model contains three anomalous $U(1)$ symmetries: $\operatorname{Tr} U_{1}=24, \operatorname{Tr} U_{2}=24, \operatorname{Tr} U_{3}=24$. Of the three anomalous $U(1) \mathrm{s}$, two can be rotated by an orthogonal transformation. One combination remains anomalous and 
is uniquely given by: $U_{A}=k \sum_{j}\left[\operatorname{Tr} U(1)_{j}\right] U(1)_{j}$, where $j$ runs over all the anomalous $U(1)$ s. The "anomalous" $U(1)_{A}$ is broken by the Dine-Seiberg-Witten mechanism [13] in which some states in the massless string spectrum obtain nonvanishing VEVs that cancel the anomalous $U(1) \mathrm{D}$-term equation. Thus, the leptophobic $U(1)_{B}$ syrnmetry is anomaly free under the entire string spectrum and can be left unbroken down to low energies.

We now examine whether similar leptophobic symmetries can arise in similar superstring models. The model of Ref. [10] differs from the model of table 1 by a change of a GSO projection coefficient. In this model, two massless gauge boson from the sector $1+\alpha+2 \gamma$ enhance one of the $U(1)$ combinations to $S U(2)_{\text {custodial }}$. The full massless spectrum and symmetries is given in Ref. [10]. However, this phase change does not modify the charges of the states from the sectors $b_{1,2,3}$ under the flavor symmetries $U(1)_{r_{4,5,6}}$. Thus, the same combination of $U(1)_{C}$ plus the flavor symmetries $U(1)_{r_{4,5,6}}$ is a leptophobic $U(1)$ symmetry. In this model the color $S U(3)$ group is not enhanced.

Next, we examine the massless spectrum of the model of Ref. [8]. The boundary condition basis vectors and the entire massless spectrum are given in Ref. [8]. The gauge boson from the NS sector are the same. The gauge group, however, is not enhanced. The sectors $b_{1,2,3}$ produce the three chiral generations, which are charged under the same flavor symmetries. The charges, however, under the flavor symmetries differ from the charges in table 1. For example, examining the charges of the states from the sector $b_{1}$,

$$
\begin{aligned}
& \left(e_{L}^{c}+u_{L}^{c}\right)_{\frac{1}{2}, 0,0, \frac{1}{2}, 0,0}+ \\
& \left(d_{L}^{c}+N_{L}^{c}\right)_{\frac{1}{2}, 0,0,-\frac{1}{2}, 0,0}+ \\
& (L)_{\frac{1}{2}, 0,0, \frac{1}{2}, 0,0}+(Q)_{\frac{1}{2}, 0,0,-\frac{1}{2}, 0,0},
\end{aligned}
$$

we observe that $e_{L}^{c}$ and $L$ have like-sign charges under $U_{r_{4}}$. Since they carry opposite sign charges under $U(1)_{C}, U_{r_{4}}$ cannot be used to cancel the $B-L$ charge for both of these states. Since, they carry like-sign charges also under $U(1)_{r_{1}}$ a leptophobic $U(1)$ cannot be made from these $U(1)$ symmetries. It also ought to be mentioned that in 
this model the flavor symmetries $U_{r 4,5,6}$ are anomalous. Therefore, their combination with $U(1)_{C}$ is not anomaly free and must be broken. Thus, the existence of a universal leptophobic $U(1)$ in the previous model is nontrivial.

Next, we comment on the charges under the horizontal symmetries in the model of Ref. [6]. This model contains as well similar horizontal flavor symmetries $U(1)_{r_{4,5,6}}$. Examining the charges of the chiral generations under these symmetries we observe that in this case the combination $U(1)_{C}+3 U(1)_{4}-3 U(1)_{5}-3 U(1)_{6}$ could serve as a leptophobic $U(1)$ symmetry. This combination cancels the $U(1)_{B-L}$ charge of the charged and doublet leptons from the sectors $b_{1,2,3}$. Interestingly, the charges of the right handed neutrinos do not vanish under this symmetry. However, in this case [6] the combination $U(1)_{5}+U(1)_{6}$ is not anomaly free and therefore cannot be a good leptophobic $U(1)$ symmetry. By changing a GSO projection coefficient we may change the sign of the charges under these symmetries, which will flip the sign of the combination. However, this is not likely to help in this model as it might also change the sign of the anomaly.

One possible interpretation of the $R_{b}$ and $R_{c}$ anomalies at LEP is the existence of an additional $Z^{\prime}$ which is universally decoupled from leptons. In this paper we examined how such leptophobic $U(1)$ symmetries may arise from superstring derived models. We showed in a specific toy model that the $U(1)_{B-L}$ gauge symmetry can combine with the horizontal flavor symmetries to produce a leptophobic $U(1)$ symmetry. The leptophobic $U(1)$ combination is universal and anomaly free. The appearance of such a symmetry seems to be nontrivial. It would be of further interest to examine whether other combinations of the flavor symmetries can produce universal leptophobic symmetries. It would be also be of interest to examine whether leptophobic $U(1)$ symmetries can arise in other classes of superstring derived models $[14,15]$. In the class of free fermionic models that we studied in this paper, the universality of the leptophobic $U(1)$ symmetry is closely related to the underlying $Z_{2} \times Z_{2}$ orbifold structure, which is exhibited in the NAHE set. Due to this underlying structure each one of the chiral generations is charged with respect to an orthogonal set of flavor quantum numbers. This property enabled a combination of the flavor $U(1)$ 
symmetries to cancel the $U(1)_{B-L}$ charge of the leptons from each sector separately, thus creating the universal leptophobic symmetry. It is also interesting to note that the appearance of a leptophobic symmetry may be correlated with proton stability in the models that we examined in some detail. In this class of models proton decay from states at the massless string level is forbidden [12]. Finally, if the LEP anomalies persist and the $Z^{\prime}$ interpretation is verified in future experiments, this discovery will indicate the existence of some structure which is beyond the Standard Model and may be beyond the simple scenarios of unification. Thus, such a discovery may be the first strong experimental evidence in favor of superstring unification, in which such additional symmetries are abundant and well motivated. It is of course important to examine in specific models whether such a leptophobic $Z^{\prime}$, which is obtained from a superstring model, can define a realistic low energy scenario. Such work is in progress.

It is a pleasure to thank Claudio Coriano and Pierre Ramond for valuable discussions. This work was supported in part by DOE Grant No. DE-FG-0586ER40272 and by CICYT under contract AEN94-0936.

\section{References}

[1] LEP Electroweak Working Group, report LEPEWWG/95-02 (August 1995).

[2] B. Holdom, Phys. Lett. B339, 114 (1994); P. Chiappetta et al, preprint PM/96-05, hep-ph/9601306; G. Altarelli et al, preprint CERN-TH/96-20, hepph/9601324; K.S. Babu, C. Kolda and J. March-Russell, preprint IASSNSHEP-96/20, hep-ph/9603212; P.H. Frampton and B.D. Wright, preprint IFP-722-UNC, hep-ph/9604260; K. Agashe et al, preprint LBL-38569, hep$\mathrm{ph} / 9604266$.

[3] A.E. Faraggi, Nucl. Phys. B387, 239 (1992).

[4] H. Kawai, D.C. Lewellen, and S.-H.H. Tye, Nucl. Phys. B288, 1 (1987); I. Antoniadis, C. Bachas, and C. Kounnas, Nucl. Phys. B289, 87 (1987). 
[5] I. Antoniadis et al, Phys. Lett. B231, 65 (1989).

[6] A.E. Faraggi, D.V. Nanopoulos, and K. Yuan, Nucl. Phys. B335, 347 (1990).

[7] I. Antoniadis, G.K. Leontaris and J. Rizos, Phys. Lett. B245, 161 (1990); G.K. Leontaris, preprint IOA.335/95, hep-ph/9601337.

[8] A.E. Faraggi, Phys. Lett. B278, 131 (1992).

[9] I. Antoniadis et al, Phys. Lett. B272, 31 (1991); J.L. Lopez, D.V. Nanopoulos and K. Yuan, Nucl. Phys. B399, 654 (1993).

[10] A.E. Faraggi, Phys. Lett. B339, 223 (1994).

[11] A.E. Faraggi, Phys. Lett. B326, 62 (1994).

[12] A.E. Faraggi, Nucl. Phys. B428, 111 (1994).

[13] M. Dine, N. Seiberg and E. Witten, Nucl. Phys. B289, 585 (1987).

[14] S. Chaudhoury, G. Hockney and J. Lykken, preprint FERMILAB-PUB-95-349$\mathrm{T}$, hep-th/9510241.

[15] L.E. Ibañez et al, Phys. Lett. B191, 282 (1987); D. Bailin, A. Love and S. Thomas, Phys. Lett. B194, 385 (1987); A. Font et al, Nucl. Phys. B331, 421 (1990). 


\begin{tabular}{|c|c|ccc|cccccccc|cccccccc|}
\hline & $\psi^{\mu}$ & $\chi^{12}$ & $\chi^{34}$ & $\chi^{56}$ & $\bar{\psi}^{1}$ & $\bar{\psi}^{2}$ & $\bar{\psi}^{3}$ & $\bar{\psi}^{4}$ & $\bar{\psi}^{5}$ & $\bar{\eta}^{1}$ & $\bar{\eta}^{2}$ & $\bar{\eta}^{3}$ & $\bar{\phi}^{1}$ & $\bar{\phi}^{2}$ & $\bar{\phi}^{3}$ & $\bar{\phi}^{4}$ & $\bar{\phi}^{5}$ & $\bar{\phi}^{6}$ & $\bar{\phi}^{7}$ & $\bar{\phi}^{8}$ \\
\hline$\alpha$ & 0 & 0 & 0 & 0 & 1 & 1 & 1 & 0 & 0 & 0 & 0 & 0 & 1 & 1 & 1 & 1 & 0 & 0 & 0 & 0 \\
$\beta$ & 0 & 0 & 0 & 0 & 1 & 1 & 1 & 0 & 0 & 0 & 0 & 0 & 1 & 1 & 1 & 1 & 0 & 0 & 0 & 0 \\
$\gamma$ & 0 & 0 & 0 & 0 & $\frac{1}{2}$ & $\frac{1}{2}$ & $\frac{1}{2}$ & $\frac{1}{2}$ & $\frac{1}{2}$ & $\frac{1}{2}$ & $\frac{1}{2}$ & $\frac{1}{2}$ & $\frac{1}{2}$ & 0 & 1 & 1 & $\frac{1}{2}$ & $\frac{1}{2}$ & $\frac{1}{2}$ & 0 \\
\hline
\end{tabular}

\begin{tabular}{|c|cccc|cccc|cccc|}
\hline & $y^{3} y^{6}$ & $y^{4} \bar{y}^{4}$ & $y^{5} \bar{y}^{5}$ & $\bar{y}^{3} \bar{y}^{6}$ & $y^{1} \omega^{6}$ & $y^{2} \bar{y}^{2}$ & $\omega^{5} \bar{\omega}^{5}$ & $\bar{y}^{1} \bar{\omega}^{6}$ & $\omega^{1} \omega^{3}$ & $\omega^{2} \bar{\omega}^{2}$ & $\omega^{4} \bar{\omega}^{4}$ & $\bar{\omega}^{1} \bar{\omega}^{3}$ \\
\hline$\alpha$ & 1 & 1 & 1 & 0 & 1 & 1 & 1 & 0 & 1 & 1 & 1 & 0 \\
$\beta$ & 0 & 1 & 0 & 1 & 0 & 1 & 0 & 1 & 1 & 0 & 0 & 0 \\
$\gamma$ & 0 & 0 & 1 & 1 & 1 & 0 & 0 & 0 & 0 & 1 & 0 & 1 \\
\hline
\end{tabular}

Table 1: A three generation $S U(4) \times S U(2) \times U(1)$ model. The choice of generalized GSO coefficients is: $c\left(\begin{array}{c}b_{j} \\ \alpha, \beta, \gamma\end{array}\right)=-c\left(\begin{array}{l}\alpha \\ 1\end{array}\right)=-c\left(\begin{array}{l}\alpha \\ \beta\end{array}\right)=-c\left(\begin{array}{l}\beta \\ 1\end{array}\right)=c\left(\begin{array}{l}\gamma \\ 1\end{array}\right)=-c\left(\begin{array}{c}\gamma \\ \alpha, \beta\end{array}\right)=$ $-1 \quad(j=1,2,3)$, with the others specified by modular invariance and space-time supersymmetry.

\section{DISCLAIMER}

This report was prepared as an account of work sponsored by an agency of the United States Government. Neither the United States Government nor any agency thereof, nor any of their employees, makes any warranty, express or implied, or assumes any legal liability or responsibility for the accuracy, completeness, or usefulness of any information, apparatus, product, or process disclosed, or represents that its use would not infringe privately owned rights. Reference herein to any specific commercial product, process, or service by trade name, trademark, manufacturer, or otherwise does not necessarily constitute or imply its endorsement, recommendation, or favoring by the United States Government or any agency thereof. The views and opinions of authors expressed herein do not necessarily state or reflect those of the United States Government or any agency thereof. 


\begin{tabular}{|c|c|c|rrrrrrr|r|rr|}
\hline$F$ & SEC & $S U(4)_{C} \times S U(2)_{L}$ & $Q_{C^{\prime}}$ & $Q_{L}$ & $Q_{1}$ & $Q_{2}$ & $Q_{3}$ & $Q_{4^{\prime}}$ & $Q_{5^{\prime}}$ & $S U(5)_{H} \times S U(3)_{H}$ & $Q_{6^{\prime}}$ & $Q_{8^{\prime \prime}}$ \\
\hline$L_{1}$ & $b_{1} \oplus$ & $(1,2)$ & $-\frac{3}{2}$ & 0 & $\frac{1}{2}$ & 0 & 0 & $-\frac{1}{2}$ & $-\frac{1}{2}$ & $(1,1)$ & $-\frac{8}{3}$ & 0 \\
$Q_{1}$ & $1+\alpha+2 \gamma$ & $(4,2)$ & $\frac{1}{2}$ & 0 & $\frac{1}{2}$ & 0 & 0 & $-\frac{1}{2}$ & $-\frac{1}{2}$ & $(1,1)$ & $-\frac{2}{3}$ & 0 \\
$d_{1}$ & & $(\overline{4}, 1)$ & $-\frac{1}{2}$ & 1 & $\frac{1}{2}$ & 0 & 0 & $\frac{1}{2}$ & $\frac{1}{2}$ & $(1,1)$ & $\frac{2}{3}$ & 0 \\
$N_{1}$ & & $(1,1)$ & $\frac{3}{2}$ & -1 & $\frac{1}{2}$ & 0 & 0 & $\frac{1}{2}$ & $\frac{1}{2}$ & $(1,1)$ & $\frac{8}{3}$ & 0 \\
$e_{1}$ & & $(1,1)$ & $\frac{3}{2}$ & 1 & $\frac{1}{2}$ & 0 & 0 & $\frac{1}{2}$ & $\frac{1}{2}$ & $(1,1)$ & $\frac{8}{3}$ & 0 \\
$u_{1}$ & & $(\overline{4}, 1)$ & $-\frac{1}{2}$ & -1 & $\frac{1}{2}$ & 0 & 0 & $\frac{1}{2}$ & $\frac{1}{2}$ & $(1,1)$ & $\frac{2}{3}$ & 0 \\
\hline$L_{2}$ & $b_{2} \oplus$ & $(1,2)$ & $-\frac{3}{2}$ & 0 & 0 & $\frac{1}{2}$ & 0 & $\frac{1}{2}$ & $-\frac{1}{2}$ & $(1,1)$ & $-\frac{8}{3}$ & 0 \\
$Q_{2}$ & $1+\alpha+2 \gamma$ & $(4,2)$ & $\frac{1}{2}$ & 0 & 0 & $\frac{1}{2}$ & 0 & $\frac{1}{2}$ & $-\frac{1}{2}$ & $(1,1)$ & $-\frac{2}{3}$ & 0 \\
$d_{2}$ & & $(4,1)$ & $-\frac{1}{2}$ & 1 & 0 & $\frac{1}{2}$ & 0 & $-\frac{1}{2}$ & $\frac{1}{2}$ & $(1,1)$ & $\frac{2}{3}$ & 0 \\
$N_{2}$ & & $(1,1)$ & $\frac{3}{2}$ & -1 & 0 & $\frac{1}{2}$ & 0 & $-\frac{1}{2}$ & $\frac{1}{2}$ & $(1,1)$ & $\frac{8}{3}$ & 0 \\
$e_{2}$ & & $(1,1)$ & $\frac{3}{2}$ & 1 & 0 & $\frac{1}{2}$ & 0 & $-\frac{1}{2}$ & $\frac{1}{2}$ & $(1,1)$ & $\frac{8}{3}$ & 0 \\
$u_{2}$ & & $(\overline{4}, 1)$ & $-\frac{1}{2}$ & -1 & 0 & $\frac{1}{2}$ & 0 & $-\frac{1}{2}$ & $\frac{1}{2}$ & $(1,1)$ & $\frac{2}{3}$ & 0 \\
\hline$L_{3}$ & $b_{3} \oplus$ & $(1,2)$ & $-\frac{3}{2}$ & 0 & 0 & 0 & $\frac{1}{2}$ & 0 & 1 & $(1,1)$ & $-\frac{8}{3}$ & 0 \\
$Q_{3}$ & $1+\alpha+2 \gamma$ & $(4,2)$ & $\frac{1}{2}$ & 0 & 0 & 0 & $\frac{1}{2}$ & 0 & 1 & $(1,1)$ & $-\frac{2}{3}$ & 0 \\
$d_{3}$ & & $(\overline{4}, 1)$ & $-\frac{1}{2}$ & 1 & 0 & 0 & $\frac{1}{2}$ & 0 & -1 & $(1,1)$ & $\frac{2}{3}$ & 0 \\
$N_{3}$ & & $(1,1)$ & $\frac{3}{2}$ & -1 & 0 & 0 & $\frac{1}{2}$ & 0 & -1 & $(1,1)$ & $\frac{8}{3}$ & 0 \\
$e_{3}$ & & $(1,1)$ & $\frac{3}{2}$ & 1 & 0 & 0 & $\frac{1}{2}$ & 0 & -1 & $(1,1)$ & $\frac{8}{3}$ & 0 \\
$u_{3}$ & & $(4,1)$ & $-\frac{1}{2}$ & -1 & 0 & 0 & $\frac{1}{2}$ & 0 & -1 & $(1,1)$ & $\frac{2}{3}$ & 0 \\
\hline
\end{tabular}

Table 2: Three generations of massless states and their quantum numbers in the model of Table 1. 


\begin{tabular}{|c|c|c|c|c|c|c|c|c|c|c|c|c|}
\hline$F$ & SEC & $S U(4)_{C} \times S U(2)_{L}$ & $Q_{C^{\prime}}$ & $Q_{L}$ & $Q_{1}$ & $Q_{2}$ & $Q_{3}$ & $Q_{4^{\prime}}$ & $Q_{5^{\prime}}$ & $S U(5)_{H} \times S U(3)_{H}$ & $Q_{6^{\prime}}$ & $Q_{8^{\prime \prime}}$ \\
\hline$V_{1}$ & $b_{1}+2 \gamma$ & $(1,1)$ & $-\frac{1}{2}$ & 0 & 0 & $\frac{1}{2}$ & $\frac{1}{2}$ & $\frac{1}{2}$ & $\frac{1}{2}$ & $(1,3)$ & $\frac{8}{3}$ & $\frac{5}{2}$ \\
\hline $\bar{V}_{1}$ & & $(1,1)$ & $\frac{1}{2}$ & 0 & 0 & $\frac{1}{2}$ & $\frac{1}{2}$ & $-\frac{1}{2}$ & $-\frac{1}{2}$ & $(1, \overline{3})$ & $-\frac{8}{3}$ & $-\frac{5}{2}$ \\
\hline$T_{1}$ & & $(1,1)$ & $\frac{1}{2}$ & 0 & 0 & $\frac{1}{2}$ & $\frac{1}{2}$ & $-\frac{1}{2}$ & $-\frac{1}{2}$ & $(5,1)$ & $-\frac{8}{3}$ & $\frac{3}{2}$ \\
\hline $\bar{T}_{1}$ & & $(1,1)$ & $-\frac{1}{2}$ & 0 & 0 & $\frac{1}{2}$ & $\frac{1}{2}$ & $\frac{1}{2}$ & $\frac{1}{2}$ & $(\overline{5}, 1)$ & $\frac{8}{3}$ & $-\frac{3}{2}$ \\
\hline$V_{2}$ & $b_{2}+2 \gamma$ & $(1,1)$ & $-\frac{1}{2}$ & 0 & $\frac{1}{2}$ & 0 & $\frac{1}{2}$ & $-\frac{1}{2}$ & $\frac{1}{2}$ & $(1,3)$ & $\frac{8}{3}$ & $\frac{5}{2}$ \\
\hline $\bar{V}_{2}$ & & $(1,1)$ & $\frac{1}{2}$ & 0 & $\frac{1}{2}$ & 0 & $\frac{1}{2}$ & $\frac{1}{2}$ & $-\frac{1}{2}$ & $(1, \overline{3})$ & $-\frac{8}{3}$ & $-\frac{5}{2}$ \\
\hline$T_{2}$ & & $(1,1)$ & $\frac{1}{2}$ & 0 & $\frac{1}{2}$ & 0 & $\frac{1}{2}$ & $\frac{1}{2}$ & $-\frac{1}{2}$ & $(5,1)$ & $-\frac{8}{3}$ & $\frac{3}{2}$ \\
\hline $\bar{T}_{2}$ & & $(1,1)$ & $-\frac{1}{2}$ & 0 & $\frac{1}{2}$ & 0 & $\frac{1}{2}$ & $-\frac{1}{2}$ & $\frac{1}{2}$ & $(\overline{5}, 1)$ & $\frac{8}{3}$ & $-\frac{3}{2}$ \\
\hline$V_{3}$ & $b_{3}+2 \gamma$ & $(1,1)$ & $-\frac{1}{2}$ & 0 & $\frac{1}{2}$ & $\frac{1}{2}$ & 0 & 0 & -1 & $(1,3)$ & $\frac{8}{3}$ & $\frac{5}{2}$ \\
\hline $\bar{V}_{3}$ & & $(1,1)$ & $\frac{1}{2}$ & 0 & $\frac{1}{2}$ & $\frac{1}{2}$ & 0 & 0 & 1 & $(1, \overline{3})$ & $-\frac{8}{3}$ & $-\frac{5}{2}$ \\
\hline$T_{3}$ & & $(1,1)$ & $\frac{1}{2}$ & 0 & $\frac{1}{2}$ & $\frac{1}{2}$ & 0 & 0 & 1 & $(5,1)$ & $-\frac{8}{3}$ & $\frac{3}{2}$ \\
\hline $\bar{T}_{3}$ & & $(1,1)$ & $-\frac{1}{2}$ & 0 & $\frac{1}{2}$ & $\frac{1}{2}$ & 0 & 0 & -1 & $(\overline{5}, 1)$ & $\frac{8}{3}$ & $-\frac{3}{2}$ \\
\hline$l_{1}$ & $b_{2}+b_{3}+$ & $(1,2)$ & $-\frac{3}{4}$ & $-\frac{1}{2}$ & $\frac{1}{4}$ & $-\frac{1}{4}$ & $-\frac{1}{4}$ & 0 & 0 & $(1,1)$ & 0 & $-\frac{15}{4}$ \\
\hline $\bar{l}_{1}$ & $\beta+\gamma+\xi$ & $(1, \overline{2})$ & $\frac{3}{4}$ & $\frac{1}{2}$ & $-\frac{1}{4}$ & $\frac{1}{4}$ & $\frac{1}{4}$ & 0 & 0 & $(1,1)$ & 0 & $\frac{15}{4}$ \\
\hline$S_{1}$ & & $(1,1)$ & $\frac{3}{4}$ & $-\frac{1}{2}$ & $-\frac{3}{4}$ & $-\frac{1}{4}$ & $-\frac{1}{4}$ & 0 & 0 & $(1,1)$ & 0 & $-\frac{15}{4}$ \\
\hline $\bar{S}_{1}$ & & $(1,1)$ & $-\frac{3}{4}$ & $\frac{1}{2}$ & $\frac{3}{4}$ & $\frac{1}{4}$ & $\frac{1}{4}$ & 0 & 0 & $(1,1)$ & 0 & $\frac{15}{4}$ \\
\hline$S_{2}$ & & $(1,1)$ & $\frac{3}{4}$ & $-\frac{1}{2}$ & $-\frac{1}{4}$ & $-\frac{3}{4}$ & $\frac{1}{4}$ & 0 & 0 & $(1,1)$ & 0 & $-\frac{15}{4}$ \\
\hline $\bar{S}_{2}$ & & $(1,1)$ & $-\frac{3}{4}$ & $\frac{1}{2}$ & $\frac{1}{4}$ & $\frac{3}{4}$ & $-\frac{1}{4}$ & 0 & 0 & $(1,1)$ & 0 & $\frac{15}{4}$ \\
\hline$S_{3}$ & & $(1,1)$ & $\frac{3}{4}$ & $-\frac{1}{2}$ & $-\frac{1}{4}$ & $\frac{1}{4}$ & $-\frac{3}{4}$ & 0 & 0 & $(1,1)$ & 0 & $-\frac{15}{4}$ \\
\hline $\bar{S}_{3}$ & & $(1,1)$ & $-\frac{3}{4}$ & $\frac{1}{2}$ & $\frac{1}{4}$ & $-\frac{1}{4}$ & $\frac{3}{4}$ & 0 & 0 & $(1,1)$ & 0 & $\frac{15}{4}$ \\
\hline$H_{1}$ & & $(1,1)$ & $-\frac{3}{4}$ & $\frac{1}{2}$ & $\frac{1}{4}$ & $-\frac{1}{4}$ & $-\frac{1}{4}$ & 0 & 0 & $(5,1)$ & 0 & $\frac{9}{4}$ \\
\hline $\bar{H}_{1}$ & & $(1,1)$ & $\frac{3}{4}$ & $-\frac{1}{2}$ & $-\frac{1}{4}$ & $\frac{1}{4}$ & $\frac{1}{4}$ & 0 & 0 & $(\overline{5}, 1)$ & 0 & $-\frac{9}{4}$ \\
\hline$l_{2}$ & $b_{1}+b_{3}+$ & $(1,2)$ & $-\frac{3}{4}$ & $-\frac{1}{2}$ & $\frac{1}{4}$ & $-\frac{1}{4}$ & $-\frac{1}{4}$ & 0 & 0 & $(1,1)$ & 0 & $-\frac{15}{4}$ \\
\hline $\bar{l}_{2}$ & $\alpha+\gamma+\xi$ & $(1, \overline{2})$ & $\frac{3}{4}$ & $\frac{1}{2}$ & $-\frac{1}{4}$ & $\frac{1}{4}$ & $\frac{1}{4}$ & 0 & 0 & $(1,1)$ & 0 & $\frac{15}{4}$ \\
\hline$S_{4}$ & & $(1,1)$ & $\frac{3}{4}$ & $-\frac{1}{2}$ & $-\frac{3}{4}$ & $-\frac{1}{4}$ & $\frac{1}{4}$ & 0 & 0 & $(1,1)$ & 0 & $-\frac{15}{4}$ \\
\hline $\bar{S}_{4}$ & & $(1,1)$ & $-\frac{3}{4}$ & $\frac{1}{2}$ & $\frac{3}{4}$ & $\frac{1}{4}$ & $-\frac{1}{4}$ & 0 & 0 & $(1,1)$ & 0 & $\frac{15}{4}$ \\
\hline$S_{5}$ & & $(1,1)$ & $\frac{3}{4}$ & $-\frac{1}{2}$ & $-\frac{1}{4}$ & $-\frac{3}{4}$ & $-\frac{1}{4}$ & 0 & 0 & $(1,1)$ & 0 & $-\frac{15}{4}$ \\
\hline $\bar{S}_{5}$ & & $(1,1)$ & $-\frac{3}{4}$ & $\frac{1}{2}$ & $\frac{1}{4}$ & $\frac{3}{4}$ & $\frac{1}{4}$ & 0 & 0 & $(1,1)$ & 0 & $\frac{15}{4}$ \\
\hline$S_{6}$ & & $(1,1)$ & $\frac{3}{4}$ & $-\frac{1}{2}$ & $\frac{1}{4}$ & $-\frac{1}{4}$ & $-\frac{3}{4}$ & 0 & 0 & $(1,1)$ & 0 & $-\frac{15}{4}$ \\
\hline $\bar{S}_{6}$ & & $(1,1)$ & $-\frac{3}{4}$ & $\frac{1}{2}$ & $-\frac{1}{4}$ & $\frac{1}{4}$ & $\frac{3}{4}$ & 0 & 0 & $(1,1)$ & 0 & $\frac{15}{4}$ \\
\hline$H_{2}$ & & $(1,1)$ & $-\frac{3}{4}$ & $\frac{1}{2}$ & $-\frac{1}{4}$ & $\frac{1}{4}$ & $-\frac{1}{4}$ & 0 & 0 & $(5,1)$ & 0 & $\frac{9}{4}$ \\
\hline $\bar{H}_{2}$ & & $(1,1)$ & $\frac{3}{4}$ & $-\frac{1}{2}$ & $\frac{1}{4}$ & $-\frac{1}{4}$ & $\frac{1}{4}$ & 0 & 0 & $(\overline{5}, 1)$ & 0 & $-\frac{9}{4}$ \\
\hline
\end{tabular}

Table 3: Extra massless states and their quantum numbers in the model of Table 1. 


\begin{tabular}{|c|c|c|rrrrrrr|c|rr|}
\hline$F$ & SEC & $S U(4)_{C} \times S U(2)_{L}$ & $Q_{C^{\prime}}$ & $Q_{L}$ & $Q_{1}$ & $Q_{2}$ & $Q_{3}$ & $Q_{4^{\prime}}$ & $Q_{5^{\prime}}$ & $S U(5)_{H} \times S U(3)_{H}$ & $Q_{6^{\prime}}$ & $Q_{8^{\prime \prime}}$ \\
\hline$l_{4}$ & $1+b_{1}+$ & $(1,2)$ & -1 & 0 & $-\frac{1}{2}$ & 0 & 0 & $-\frac{1}{2}$ & $-\frac{1}{2}$ & $(1,1)$ & $\frac{16}{3}$ & 0 \\
$S_{7}$ & $\alpha+2 \gamma$ & $(1,1)$ & 1 & 1 & $-\frac{1}{2}$ & 0 & 0 & $\frac{1}{2}$ & $\frac{1}{2}$ & $(1,1)$ & $-\frac{16}{3}$ & 0 \\
$\bar{S}_{7}$ & & $(1,1)$ & 1 & -1 & $-\frac{1}{2}$ & 0 & 0 & $\frac{1}{2}$ & $\frac{1}{2}$ & $(1,1)$ & $-\frac{16}{3}$ & 0 \\
\hline$l_{5}$ & $1+b_{2}+$ & $(1,2)$ & -1 & 0 & 0 & $-\frac{1}{2}$ & 0 & $\frac{1}{2}$ & $-\frac{1}{2}$ & $(1,1)$ & $\frac{16}{3}$ & 0 \\
$S_{8}$ & $\alpha+2 \gamma$ & $(1,1)$ & 1 & 1 & 0 & $-\frac{1}{2}$ & 0 & $-\frac{1}{2}$ & $\frac{1}{2}$ & $(1,1)$ & $-\frac{16}{3}$ & 0 \\
$\bar{S}_{8}$ & & $(1,1)$ & 1 & -1 & 0 & $-\frac{1}{2}$ & 0 & $-\frac{1}{2}$ & $\frac{1}{2}$ & $(1,1)$ & $-\frac{16}{3}$ & 0 \\
\hline$l_{6}$ & $1+b_{3}+$ & $(1,2)$ & -1 & 0 & 0 & 0 & $-\frac{1}{2}$ & 0 & 1 & $(1,1)$ & $\frac{16}{3}$ & 0 \\
$S_{9}$ & $\alpha+2 \gamma$ & $(1,1)$ & 1 & 1 & 0 & 0 & $-\frac{1}{2}$ & 0 & -1 & $(1,1)$ & $-\frac{16}{3}$ & 0 \\
$\bar{S}_{9}$ & & $(1,1)$ & 1 & -1 & 0 & 0 & $-\frac{1}{2}$ & 0 & -1 & $(1,1)$ & $-\frac{16}{3}$ & 0 \\
\hline$S_{10}$ & $1+s+$ & $(1,1)$ & -2 & 0 & 0 & 0 & 0 & -1 & -1 & $(1,1)$ & $-\frac{4}{3}$ & 0 \\
$\bar{S}_{10}$ & $\alpha+2 \gamma$ & $(1,1)$ & -2 & 0 & 0 & 0 & 0 & 1 & 1 & $(1,1)$ & $\frac{4}{3}$ & 0 \\
\hline
\end{tabular}

Table 4: (Cont.) Extra massless states and their quantum numbers in the model of Table 1. 